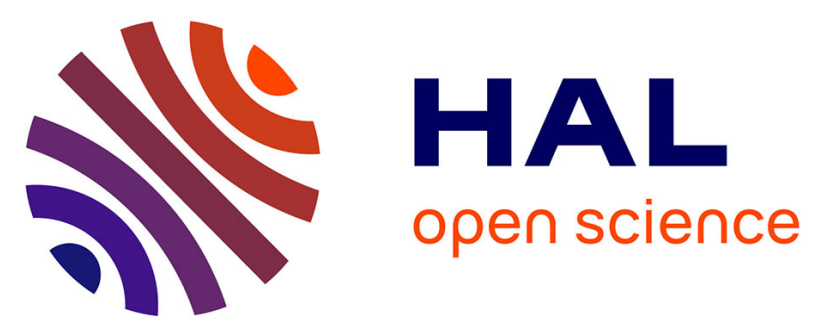

\title{
ÉVIDENCE D'UN DÉSORDRE A MOYENNE DISTANCE DANS LES SOLIDES. INTERPRÉTATION DES PROPRIÉTÉS ÉLECTRIQUES DES SEMICONDUCTEURS DÉSORDONNÉS
}

B. Pistoulet, J. Robert, J.-M. Dusseau, F. Roche, Pascal Girard

\section{To cite this version:}

B. Pistoulet, J. Robert, J.-M. Dusseau, F. Roche, Pascal Girard. ÉVIDENCE D'UN DÉSORDRE A MOYENNE DISTANCE DANS LES SOLIDES. INTERPRÉTATION DES PROPRIÉTÉS ÉLECTRIQUES DES SEMICONDUCTEURS DÉSORDONNÉS. Journal de Physique Colloques, 1977, 38 (C7), pp.C7-207-C7-210. 10.1051/jphyscol:1977739 . jpa-00217240

HAL Id: jpa-00217240 https://hal.science/jpa-00217240

Submitted on 1 Jan 1977

HAL is a multi-disciplinary open access archive for the deposit and dissemination of scientific research documents, whether they are published or not. The documents may come from teaching and research institutions in France or abroad, or from public or private research centers.
L'archive ouverte pluridisciplinaire HAL, est destinée au dépôt et à la diffusion de documents scientifiques de niveau recherche, publiés ou non, émanant des établissements d'enseignement et de recherche français ou étrangers, des laboratoires publics ou privés. 


\title{
EVIDENCE D'UN DÉSORDRE A MOYENNE DISTANCE DANS LES SOLIDES. INTERPRÉTATION DES PROPRIÉTÉS ÉLECTRIQUES DES SEMICONDUCTEURS DÉSORDONNÉS
}

\author{
B. PISTOUleT, J. L. ROBERT, J. M. DUSSEAU, F. ROCHE et P. GIRARD \\ Centre d'Etudes d'Electronique des Solides $\left(^{*}\right)$, Université des Sciences et Techniques du Languedoc, \\ Montpellier, France
}

\begin{abstract}
Résumé. - On montre la nécessité de considérer l'existence d'un désordre à moyenne distance dans les semiconducteurs désordonnés. Il provoque la localisation d'une partie des porteurs dans les puits de potentiel, et explique quantitativement les variations de la mobilité d'entraînement et de la conductivité électrique avec la température et la fréquence.
\end{abstract}

\begin{abstract}
The necessity of considering a medium range disorder in disordered semiconductors is shown. This disorder is responsible for the localization, in potential wells, of a part of the total carriers density, and explains quantitatively the variation's of drift mobility and electrical conductivity versus temperature and frequency.
\end{abstract}

1. Introduction. - Les propriétés des solides sont généralement étudiées dans deux hypothèses extrêmes : s'il s'agit de monocristaux, l'ordre est supposé quasi parfait, et la distribution des impuretés et des défauts est supposée homogène; dans le cas de solides désordonnés ou amorphes on considère un désordre microscopique à l'échelle interatomique, qui se traduit par un potentiel variable d'un site atomique à un autre, et conduit aux modèles d'Anderson et de MottCohen-Fritzsche-Ovshinsky [1-4]. Il faut remarquer que dans ces théories, le matériau est toujours considéré comme macroscopiquement homogène.

Nous proposons, dans cet article, une interprétation nouvelle des phénomènes observés dans les semiconducteurs désordonnés, basée sur l'existence d'un désordre à moyenne distance dans ces matériaux. Nous avons précédemment mis en évidence ce désordre de distribution spatiale des impuretés dans les monocristaux semiconducteurs [5], et montré son influence sur l'ensemble des phénomènes de transport $[6,7]$. Selon ce modèle, la concentration d'impuretés varie lentement d'un point à un autre du spécimen qui peut donc être considéré comme constitué de domaines de dopage uniforme, mais variable d'un domaine à l'autre. Pour être mises en évidence par des expériences de transport, les dimensions des domaines doivent être au moins égales au libre parcours moyen et à la longueur d'écran de Debye. La formation de

(*) Laboratoire associé au C.N.R.S. ces domaines, au cours de la croissance du cristal, est provoquée par des accidents inévitables tels que fluctuations de température, gradients thermiques, variations de débits gazeux ou liquides, courants de convection, etc...

Les fluctuations spatiales de densité d'impuretés, et éventuellement de stochiométrie, sont certainement plus importantes dans les matériaux désordonnés, et le désordre à moyenne distance y provoque deux effets principaux : d'une part les mesures représentent, non les valeurs des grandeurs physiques en chaque point du matériau, mais une moyenne sur l'ensemble des domaines; d'autre part, nous montrons que ce désordre provoque un nouveau phénomène, la localisation d'une fraction des porteurs de la bande. Ces deux effets permettent de rendre compte quantitativement des phénomènes de transport observés.

2. Modèle proposé pour décrire un semiconducteur désordonné. - Nous raisonnerons sur un semiconducteur de type n non dégénéré.

2.1 Fluctuations SPatiales de $E_{\mathrm{c}}$ - - Les variations de la densité d'impuretés, ou plus généralement de la composition, se traduisent par des variations de l'énergie $E_{\mathrm{c}}-E_{\mathrm{F}}$, où $E_{\mathrm{c}}$ est l'énergie potentielle en bas de bande et $E_{\mathrm{F}}$ le niveau de Fermi. 
A l'équilibre, l'énergie $E_{\mathrm{c}}$ obéit, dans l'échantillon, à une loi de probabilité que nous écrirons :

$$
\left.\begin{array}{l}
P\left(E_{\mathrm{c}}\right)=\frac{1}{\Gamma \sqrt{\Pi}} \exp \left[-\left(\frac{E_{\mathrm{c}}-E_{\mathrm{c} 0}}{\Gamma}\right)^{2}\right], \text { pour }\left|\frac{E_{\mathrm{c}}-E_{\mathrm{c} 0}}{\Gamma}\right|<g \\
P\left(E_{\mathrm{c}}\right)=0 \text { pour }\left|\frac{E_{\mathrm{c}}-E_{\mathrm{c} 0}}{\Gamma}\right|>g ; \text { où } g \simeq 3
\end{array}\right\}
$$

$E_{\mathrm{c} 0}$ est la valeur la plus probable de $E_{\mathrm{c}}$ au sein de l'échantillon et $\Gamma$ le paramètre de désordre; la gaussienne est limitée à l'intervalle $-g,+g$ de façon à éviter de prendre en considération des écarts très grands, et de signification physique douteuse, et assurer la non-dégénérescence de tous les domaines (la correction à apporter à la norme est négligeable).

2.2 Localisation Des PORTeURS Dans Les pUits DE POTENTIEL. - Les variations de $E_{\mathrm{c}}$ se traduisent par l'existence de puits de potentiel, plus ou moins profonds, disséminés dans le matériau, dans lesquels une fraction des porteurs de la bande, d'autant plus grande que $\Gamma / k T$ est grand, se trouve localisée; seuls les porteurs d'énergie égale ou supérieure à $E_{\mathrm{c} 0}-\alpha k T$ (avec $0 \leqslant \alpha \leqslant 3$ ) sont libres de se déplacer dans la bande. Ce phénomène essentiel, qui n'avait jamais été pris en considération jusqu'ici, est tout à fait différent d'un piégeage sur des niveaux de la bande interdite. Soit $n$ la densité totale de porteurs dans la bande, $n_{1}$ la densité de poteurs libres; les $n-n_{1}$ porteurs localisés ne participent pas à la conduction en continu.

2.3 EXPRESSION DE $\mu_{\mathrm{D}}, \mu_{\mathrm{H}}$ ET $\sigma$ EN COURANT CONTINU. - La mobilité d'entraînement $\mu_{\mathrm{D}}$, et la conductivité en continu $\sigma$ sont reliées [8] à la mobilité $\mu_{0}$ en l'absence de piégeage, ou ici de localisation, par :

$$
\begin{aligned}
\mu_{\mathbf{D}} & =\frac{n_{1}}{n} \mu_{0} \\
\sigma & =n q \mu_{\mathbf{D}}=n_{1} q \mu_{0} .
\end{aligned}
$$

Seuls les porteurs libres contribuent à l'effet Hall, comme nous l'avons montré dans les domaines acoustoélectriques [10], de sorte que l'on exprime aisément la mobilité de Hall $\mu_{\mathbf{H}}$, qui a été déterminée expérimentalement sur différents corps [9], en fonction de $\mu_{\mathbf{D}}$ :

$$
\mu_{\mathbf{H}}=R_{\mathbf{H}} \sigma=\mu_{0}=\mu_{\mathrm{D}} \frac{n}{n_{1}}>\mu_{\mathrm{D}}
$$

2.4 ETATS LOCALISÉS DANS LA BANDE INTERDITE. - Le désordre à courte distance et les impuretés introduisent des états localisés dans la bande interdite ; on considère généralement que ces états forment un continuum. Remarquons que, d'après notre modèle, chaque niveau discret situé à une énergie $E_{\mathrm{c}}-E_{\mathrm{d}}$ donne naissance, lorsqu'on considère l'effet moyen dû à tous les domaines, à une densité apparente d'états de la forme (1) mais centrée sur l'énergie $E_{\mathrm{c} 0}-E_{\mathrm{d}}$, et que, de même, la limite $E_{\mathrm{c}}$ de la bande de conduction donne naissance, par cet effet de moyenne, à une queue de bande dont la signification physique est très différente de celle généralement admise. On peut donc rendre compte de la densité d'états localisés dans le gap au moyen d'un nombre très limité de niveaux. Le modèle le plus simple, considéré ici, comporte un niveau donneur dominant profond, d'énergie d'ionisation $E_{\mathrm{d}}$ et de densité $N_{\mathrm{d}}$, et des niveaux accepteurs de densité totale $N_{\mathrm{a}}$.

3. Lois $\sigma(T)$ et $\mu_{\mathrm{D}}(T)$ imposées par le désordre à moyenne distance. - $\mathrm{Si} \mu_{0}(T)$ est connue, la détermination de $\sigma$ et $\mu_{\mathrm{D}}$ se ramène au calcul de $n_{1}$ et $n$. Soit $E$ l'énergie d'un porteur; nous poserons :

$$
\begin{array}{cr}
\varepsilon=\frac{E-E_{\mathrm{c} 0}}{k T} ; \quad x=\frac{E_{\mathrm{c} 0}-E_{\mathrm{c}}}{k T} ; \\
\eta=\frac{E_{\mathrm{F}}-E_{\mathrm{c}}}{k T} ; \quad \eta_{\mathrm{o}}=\frac{E_{\mathrm{F}}-E_{\mathrm{c} 0}}{k T} .
\end{array}
$$

La densité de porteurs dans un domaine s'écrit :

$$
n(x)=N_{\mathrm{c}} \mathrm{e}^{\eta}=n_{0} \mathrm{e}^{x}, \text { avec } n_{0}=N_{\mathrm{c}} \mathrm{e}^{\eta_{0}} \text {. }
$$

Dans un matériau assez compensé, et si $E_{\mathrm{d}} / k T \gg 1$, $n_{0}$ s'écrit [11] :

$$
n_{0}=\frac{N_{\mathrm{c}}}{2}\left(\frac{N_{\mathrm{d} 0}}{N_{\mathrm{a} 0}}-1\right) \mathrm{e}^{-E_{\mathrm{d}} / k T} .
$$

On obtient la densité totale de porteurs $n$ à l'aide de la loi de probabilité (1) exprimée en fonction de $x$ :

$$
n=\int_{-g \Gamma / k T}^{g \Gamma / k T} n(x) P(x) \mathrm{d} x=\frac{N_{\mathrm{c}}}{2 \sqrt{\Pi}}\left(\frac{N_{\mathrm{d} 0}}{N_{\mathrm{a} 0}}-1\right) \exp \left(-\frac{E_{\mathrm{d}}}{k T}+\frac{\Gamma^{2}}{4 k^{2} T^{2}}\right) \int_{0}^{2 g} \exp \left[-\left(v-g-\frac{\Gamma}{2 k T}\right)^{2}\right] \mathrm{d} v .
$$

La densité $n_{1}$ de porteurs libres est la somme de la densité $n_{11}$ de porteurs dans les domaines où $x<\alpha$, et de la densité $n_{21}$ de porteurs d'énergie $E>E_{\mathrm{co}}$ provenant des domaines où $x>\alpha$ : 


$$
\begin{aligned}
n_{1} & =n_{11}+n_{21} \\
n_{11} & =\frac{N_{\mathrm{c}}}{2 \sqrt{\Pi}}\left(\frac{N_{\mathrm{d} 0}}{N_{\mathrm{a} 0}}-1\right) \exp \left(-\frac{E_{\mathrm{d}}}{k T}+\frac{\Gamma^{2}}{4 k^{2} T^{2}}\right) \int_{0}^{g+(\alpha k T / \Gamma)} \exp \left[-\left(v-g-\frac{\Gamma}{2 k T}\right)^{2}\right] \cdot \mathrm{d} v \\
n_{21} & =\frac{N_{\mathrm{c}}}{\Pi}\left(\frac{N_{\mathrm{d} 0}}{N_{\mathrm{a} 0}}-1\right) \exp \left(\alpha-\frac{E_{\mathrm{d}}}{k T}\right) \int_{\alpha k T / \Gamma}^{g} \mathrm{e}^{-v^{2}} \int_{0}^{\infty}\left(u-\alpha+\frac{\Gamma v}{k T}\right)^{1 / 2} \cdot \mathrm{e}^{-u} \mathrm{~d} u \cdot \mathrm{d} v .
\end{aligned}
$$

Dans la suite nous prendrons $\alpha=0$.

4. Interprétation de résultats expérimentaux. - On détermine d'abord le paramètre de désordre $\Gamma$ en essayant, par approximations successives, de rendre compte, au mieux, de la courbe expérimentale $\mu_{\mathrm{D}}(T)$ à l'aide de (3), (9), (10). On détermine ensuite $E_{\mathrm{d}}$ en comparant les valeurs expérimentales de $\sigma / \mu_{\mathbf{D}}=q n$ et (9); aux températures élevées, le terme $\exp \left(\Gamma^{2} / 4 k^{2} T^{2}\right)$ est voisin de l'unité et la pente de la courbe $\log n=f(1 / k T)$ est pratiquement égal à $-E_{\mathrm{d}}$.

Sur les figures 1 et 2 on a reporté la variation de $\mu_{\mathbf{D}}(T)$ trouvée par D. M. Pai [9] et celle de $\sigma$ donnée par G. Juska [9] pour le sélénium, ainsi que les courbes calculées au moyen de (3), (4), (11), (12), en prenant $E_{\mathrm{d}}=0,80 \mathrm{eV}, \Gamma=0,15 \mathrm{eV}$. Sur la figure 3 sont reportées les valeurs de la mobilité mesurée par Main et Owen [9] sur $\mathrm{As}_{2} \mathrm{Te}_{3}$ amorphe; on obtient un bon accord pour $\Gamma=0,085 \mathrm{eV}$. Les courbes $\mu_{\mathrm{D}}(T)$ et $\sigma(T)$ obtenues par Marshall et Owen [12] sur $\mathrm{As}_{2} \mathrm{Te}_{3}$ sont reportées sur les figures 3 et 4 ; les valeurs de $\mu_{\mathrm{H}}(T)$ sont dues à Grant et al. et Roilos et al. [9]; on en déduit $E_{\mathrm{d}}=0,36 \mathrm{eV}$, et $\Gamma=0,082 \mathrm{eV}$. Le désaccord observé à basse température provient très probablement du fait que la conductivité étant alors très faible, les mesures ont probablement été effectuées

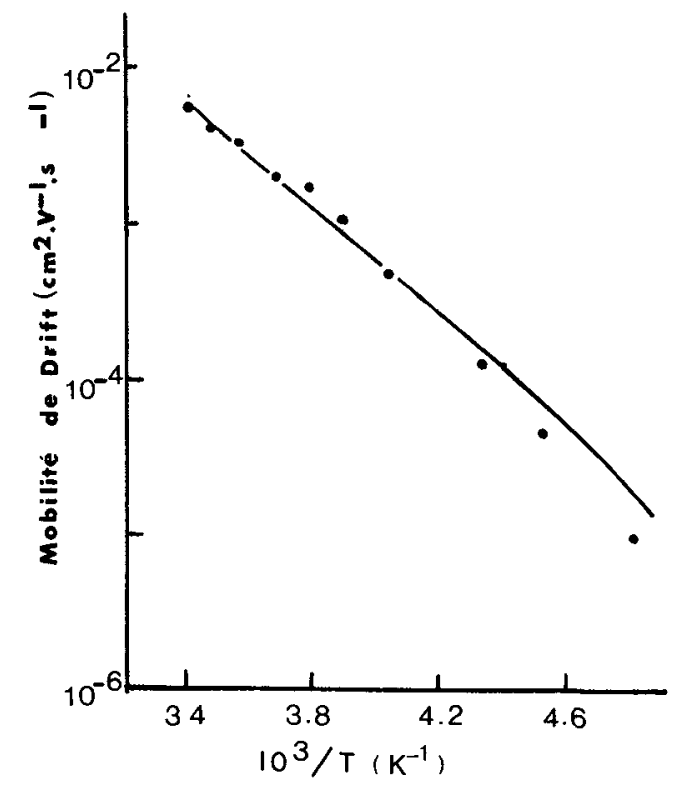

FIG. $1 .-\mu_{\mathrm{D}}(T)$ dans Se. Points : D. M. Pai, $\longrightarrow$ courbe calculée par (3). à champ électrique plus élevé ; or $\mu_{\mathrm{D}}$ et $\sigma$ croissent avec le champ électrique $[12,13]$. L'étude détaillée des effets à forts champs, sort du cadre du présent article, et sera prochainement publiée par ailleurs. Enfin les résultats obtenus [12] sur $\mathrm{As}_{30} \mathrm{Te}_{48} \mathrm{Si}_{12} \mathrm{Ge}_{10}$ sont correctement interprétés en prenant $E_{\mathrm{d}}=0,50 \mathrm{eV}$ et $\Gamma=0,15 \mathrm{eV}$.

5. Autres propriétés expliquées par le modèle de désordre à moyenne distance. - La croissance de la conductivité avec la fréquence signalée par Stourac [9] et Rokstad [14] pourrait résulter du fait que, lorsque la fréquence croît, une fraction de plus en plus importante des porteurs localisés participe à la conduction : ce sont ceux qui effectuent, en une demi-période, un trajet inférieur à la dimension du puits de potentiel; les électrons au fond des puits ne conduisent qu'à des fréquences très élevées, et alors le nombre apparent de porteurs libres tend vers $n$.

Stourac [9] et d'autres auteurs, ont montré que la permittivité peut être très grande $\left(\varepsilon_{\mathrm{r}}>10^{3}\right)$ en basse fréquence et qu'elle décroît rapidement et tend,

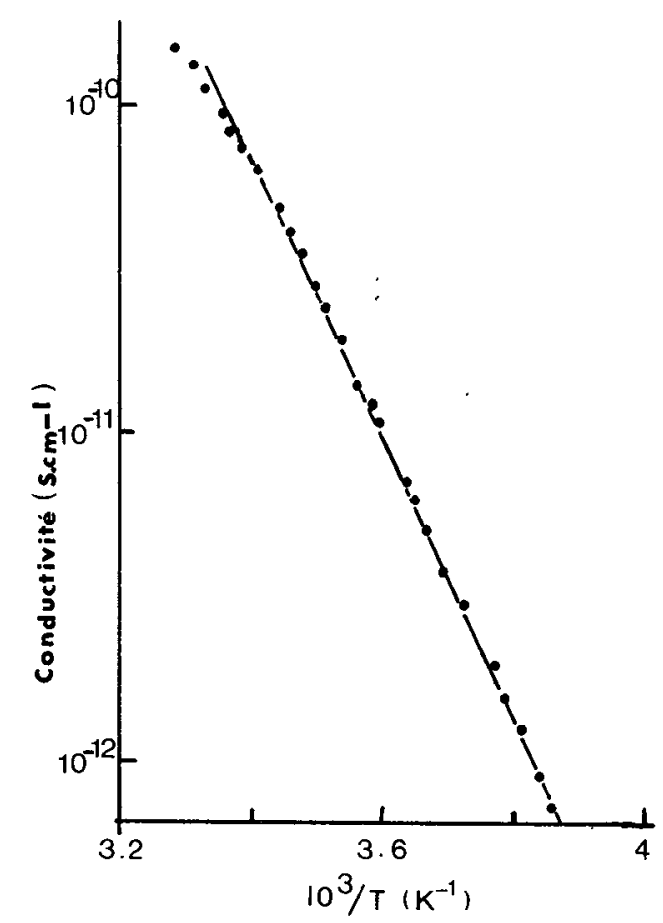

FIG. 2. $-\sigma(T)$ dans Se. Points : G. Juska, - courbe calculée par (9). 


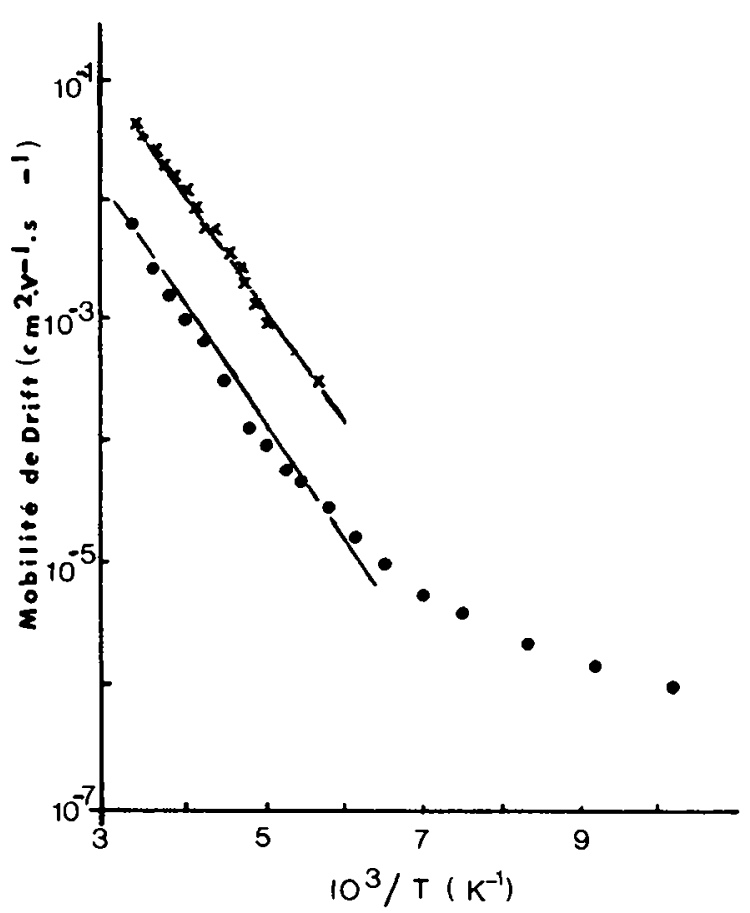

FIG. 3. $-\mu_{\mathrm{D}}(T)$ dans $\mathrm{As}_{2} \mathrm{Te}_{3}:+$ Main et Owen; et Owen, - courbes calculées par (3).

au-dessus de $10^{6}$ à $10^{8} \mathrm{~Hz}$ vers une limite de l'ordre de $\varepsilon_{\mathrm{r}}=10$. Cet effet serait accru par la présence des porteurs localisés qui, sous l'action du champ, provoquent l'apparition de $n-n_{1}(\omega)$ dipôles, donc d'une permittivité supplémentaire proportionnelle à $n-n_{1}(\omega)$. Cette permittivité peut être très grande en B.F. et tend vers zéro en H.F.

Enfin, il est clair qu'un matériau peut, ou non, être photoconducteur suivant que $\Gamma$ est petit ou grand.

6. Conclusion. - Nous avons montré que, dans les composés désordonnés, le désordre à moyenne distance joue un rôle essentiel, même à température élevée. Ce phénomène, ignoré jusqu'ici, entraîne la localisation d'une partie importante des porteurs de la bande. Le modèle proposé permet de rendre compte quantitativement des phénomènes observés dans les semiconducteurs désordonnés, vitreux, amorphes et

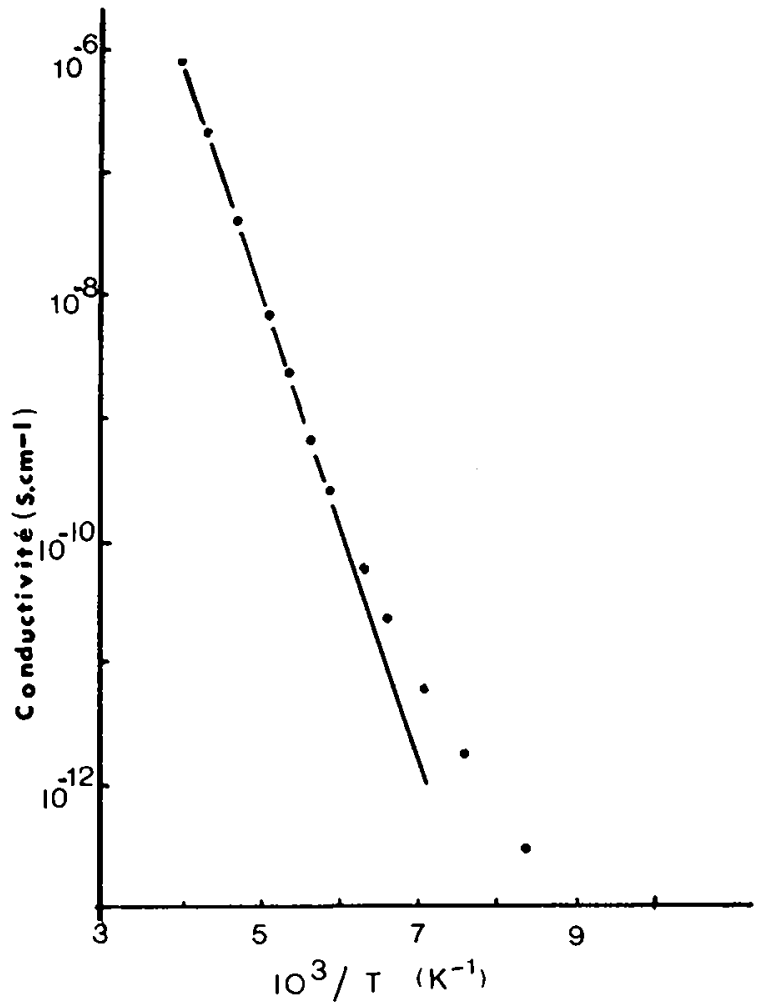

FiG. 4. $-\sigma(T)$ dans $\mathrm{As}_{2} \mathrm{Te}_{3}$ : Marshall et Owen, — courbe calculée par (9)

sans doute aussi dans les liquides et les isolants. A condition de considérer un domaine unique, le modèle physique n'est pas fondamentalement différent de celui qui s'applique aux monocristaux. La différence essentielle réside dans l'ordre de grandeur du désordre à moyenne distance. Quant au désordre à très courte distance, son rôle dans les phénomènes de transport apparaît moins important qu'on ne le pense généralement.

Enfin, nous pensons que cette description du désordre dans un solide est susceptible de s'appliquer de façon plus générale, à des matériaux autres que les semiconducteurs, à condition que les fluctuations spatiales de composition de ces matériaux entraînent des variations de la valeur moyenne d'une grandeur physique dans l'échantillon.

\section{Bibliographie}

[1] Mort, N. F. and Davies, E. A., Electronic Process in noncrystalline materials (Clarendon Press, Oxford) 1971.

[2] Eggarter, T P., Phys. Rev. A 5 (1972) 2496.

[3] Fritsche, H., J. Non-Cryst. Solids 6 (1971) 49.

[4] Anderson, P. W., Phys. Rev. Lett. 34 (1975).

[5] Pistoulet, B., Robert, J. L.. Raymond, A., Barjon, D., Joullie, A., Solid State Commun. 16 (1975) 289.

[6] Raymond, A., Robert, J. L., Pistoulet, B., Proc. GaAs and related compounds Conf. Edinburgh, 1976, p. 105.

[7] Raymond, A., Robert, J. L. et al., III Internat. Conf. Phys. narrow gap semicond., Warshaw 1977.

[8] Shocklfy, W., Electrons and Holes in semiconductors (Van Nostrand) 1960, p. 211
[9] Proc. 5th Int. Conf. Amorphous and liquid Semicond. Garmish 1973 (Taylor and Francis) : Grant, A. J. et al., p. 325 ; RoILOs, M. et al., p. 319 ; PAI, D. et al., p. 355 ; MAIN, C., OWfin, A. E., p. 783 ; Stourac, L. et al., p. 29 ; Juska, G. et al., p. 363 .

[10] Lepètre, T., Dusseau, J. M., Robert, J. L., Pistoulet, B., J. Phys. C 6 (1794) 1973.

[11] Blakemore, J., Semiconductors Statistics (Pergamon Press) $1962 ;$ p. 135.

[12] Marshall, T. M., Owen, A. E., Phil. Mag. 31 (1975) 1341 ; ibid 24 (1971) 1281 .

[13] Marshall, J. M., Main, C., Owen, A. E., J. Non-Cryst. Solids 8-10 (1972) 760 .

[14] Rockstad, H. K., J. Non-Cryst. Solids 8-10 (1972) 621. 